\title{
Selective Medium for Isolation of Clostridium butyricum from Human Feces
}

\author{
M. R. POPOFF \\ Service des Anaérobies, Institut Pasteur, 75724 Paris Cedex 15, France
}

Received 8 February 1984/Accepted 4 June 1984

\begin{abstract}
A selective medium, Clostridium butyricum isolation medium (BIM), is described for the isolation of $C$. butyricum from human feces. The BIM is a synthetic minimal medium and contains trimethoprim $(16 \mu \mathrm{g} / \mathrm{ml})$, D-cycloserine $(10 \mu \mathrm{g} / \mathrm{ml})$, and polymyxin B sulfate $(20 \mu \mathrm{g} / \mathrm{ml})$ as selective inhịbitory agents. Qualitative tests indicated that $C$. butyricum and other butyric acid-producing clostridia grew on BIM, Clostridium sphenoides and Bacillus cereus produced small colonies, and other clostridia and other obligate anaerobic or facultatively anerobic bacteria were inhibited. Quantitative recovery of $C$. butyricum from cultures or seeded fecal samples was comparable with BIM and with complex medium, but the quantitative recovery of the other butyric acidproducing clostridia tested (C. beijerinckii, $C$. acetobutylicum) was lower with BIM than with complex medium. The BIM should aid the rapid isolation of $C$. butyricum from fecal samples and should be useful for bacteriological investigation of neonatal necrotizing enterocolitis.
\end{abstract}

Clostridium butyricum has been incriminated in some cases of neonatal necrotizing enterocolitis $(8,10,15,19)$. This organism was present at high levels in the feces of these infants. However, $C$. butyricum was also recovered from stools of normal newborns. Neonatal necrotizing enterocolitis has multifactorial origin, and bacteria, especially clostridia, seem to play a central role in the initiation of intestinal lesions.

Qualitative and quantitative bacteriological examinations of fecal specimens are important methods for neonatal necrotizing enterocolitis etiological investigation. $C$. butyricum isolation is usually done by employing nonselective media designed for the recovery of clostridia. In a previous investigation of experimental infection in guinea pigs with $C$. butyricum, I recognized the need to use a selective medium for quantitative evaluation of this organism in intestinal content (manuscript in preparation). On the basis of the minimal medium for $C$. butyricum described by Cummins and Johnson (2), I have developed a selective medium for the isolation of $C$. butyricum from feces.

\section{MATERIALS AND METHODS}

Bacteria. I used 255 strains of bacteria: 34 strains of $C$. butyricum, 10 strains of Clostridium beijerinckii, 126 strains (39 species) of other clostridia, 24 strains (14 species) of nonspore-forming anaerobes, and 55 strains (52 species) of various facultatively anaerobic bacteria. $C$. butyricum and C. beijerinckii strains were identified by the method previously described (13). For each species of Clostridium, the type strain listed in the Approved Lists (17) and obtained from the American Type Culture Collection was included. The other strains of clostridia and of non-spore-forming anaerobes were identified in our laboratory according to the manual of Holdeman et al. (7). The facultatively anaerobic bacteria were obtained from various reference laboratories at the Pasteur Institute.

Isolation medium for $C$. butyricum. The basal medium used by Cummins and Johnson (2) for determining the nutritional requirements of $C$. butyricum was employed with some modifications of the buffer solution. Several antimicrobial agents in various concentrations were tested. The better results, allowing growth of $C$. butyricum and inhibition of other fecal bacteria, were obtained with trimethoprim, Dcycloserine, and polymyxin B sulfate (all from Sigma Chemical Co.). The concentration of each antimicrobial agent was defined in a preliminary experiment (see Table 1 ). The $C$. butyricum isolation medium (BIM) had the following composition: concentrated mineral salts solution, $100 \mathrm{ml}$; phosphate solution, $100 \mathrm{ml}$; iron sulfate $(0.1 \%$ aqueous solution), $10 \mathrm{ml}$; biotin $(0.002 \%$ aqueous solution), $0.25 \mathrm{ml}$; cysteinehydrochloride, $0.5 \mathrm{~g}$; glucose, $10 \mathrm{~g}$; agar, $15 \mathrm{~g}$; trimethoprim, $16 \mathrm{mg}$; cycloserine, $10 \mathrm{mg}$; polymyxin $\mathrm{B}$ sulfate, $20 \mathrm{mg}$;

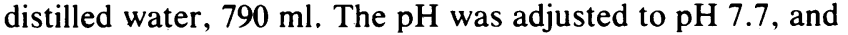
the medium was autoclaved at $110^{\circ} \mathrm{C}$ for $20 \mathrm{~min}$.

The concentrated mineral salts solution contained: $\mathrm{NaCl}$, $9 \mathrm{~g} ; \mathrm{CaCl}_{2}, 200 \mathrm{mg} ; \mathrm{MgCl}_{2} \cdot 6 \mathrm{H}_{2} \mathrm{O}, 200 \mathrm{mg} ; \mathrm{MnCl}_{2} \cdot 4 \mathrm{H}_{2} \mathrm{O}$, $100 \mathrm{mg}$; $\mathrm{Cocl}_{2} \cdot 6 \mathrm{H}_{2} \mathrm{O}, 10 \mathrm{mg}$; distilled water, $1,000 \mathrm{ml}$. The phosphate solution contained: $\mathrm{KH}_{2} \mathrm{PO}_{4}, 7 \mathrm{~g} ; \mathrm{K}_{2} \mathrm{HPO}_{4}, 7 \mathrm{~g}$; distilled water, $100 \mathrm{ml}$. The antimicrobial agents were added to the medium after it was autoclaved and cooled to $50^{\circ} \mathrm{C}$ in a water bath. The stock solutions of $\mathrm{D}$-cycloserine $(0.25 \%)$ and polymyxin B sulfate $(0.25 \%)$ were made in distilled water. The trimethoprim stock solution $(0.1 \%)$ was made at $55^{\circ} \mathrm{C}$ distilled water acidified with $0.05 \mathrm{~N} \mathrm{HCl}$ as described by Dezfulian et al. (3). Sixteen milliliters of trimethoprim, $4 \mathrm{ml}$ of the cycloserine, and $8 \mathrm{ml}$ of the polymyxin $B$ sulfate solution were added to 1 liter of medium.

Fluid basal medium (FBM), prepared as BIM without agar, glucose, and antimicrobial agents, was used for fecal specimen dilutions. In some experiments, we used a basal medium (BM) that was BIM without antimicrobial agents.

Evaluation of BIM. The growth of various bacteria was studied on BIM and on a complex meat-yeast extract medium (VL) (1). Overnight cultures in Trypticase (BBL Microbiology Systems)-yeast extract-glucose broth (16) were diluted 1:10 in FBM and streaked on BIM and VL plates. The plates were incubated for $48 \mathrm{~h}$ at $37^{\circ} \mathrm{C}$ in an anaerobic glove box flushed with $\mathrm{H}_{2}-\mathrm{CO}_{2}, 95: 5(\mathrm{vol} / \mathrm{vol})$. For the quantitative recovery of $C$. butyricum strains on BIM and VL media, dilutions in FBM were made in an anaerobic chamber (La 
Calhène) containing $\mathrm{N}_{2}-\mathrm{CO}_{2}-\mathrm{H}_{2}, 80: 15: 5(\mathrm{vol} / \mathrm{vol})$; then 0.1 $\mathrm{ml}$ of $10^{3}, 10^{4}$, and $10^{5}$ dilutions was spread over each plate. The colonies were counted after plate incubation for $48 \mathrm{~h}$ at $37^{\circ} \mathrm{C}$ in an anaerobic chamber. $C$. butyricum spores, obtained in D medium (9), were enumerated on BIM and VL plates after heat treatment of the culture at $80^{\circ} \mathrm{C}$ for $10 \mathrm{~min}$.

BIM was also tested for $C$. butyricum recovery from seeded fecal specimens. A 1-g human fecal sample was homogenized in $3 \mathrm{ml}$ of FBM. Then 1 volume of a $1: 10$ dilution of the overnight $C$. butyricum cell suspension in Trypticase-yeast extract-glucose broth was mixed with 4 volumes of fecal suspension and $0.1 \mathrm{ml}$ of $10^{1}, 10^{2}$, and $10^{3}$ dilutions in FBM was spread over the surface of BIM plates. All manipulations were done in an anaerobic chamber. The colonies were counted after $48 \mathrm{~h}$ of incubation at $37^{\circ} \mathrm{C}$. Some colonies of each plate were examined for their microscopic appearances and tested for sugar fermentation. The number of $C$. butyricum colonies thus recovered from a fecal sample on BIM was compared with the number of viable bacteria added to the fecal sample, as counted on VL plates.

\section{RESULTS}

Concentrations of antimicrobial agents in BIM. The quantitative recovery of $C$. butyricum on VL medium and on BM with various concentrations of trimethoprim, cycloserine, and polymyxin B is shown in Table 1 . Based on these data and data from several similar experiments, I decided to use $16 \mu \mathrm{g}$ of trimethoprim, $10 \mu \mathrm{g}$ of cycloserine, and $20 \mu \mathrm{g}$ of $\mathrm{B}$ polymyxin per $\mathrm{ml}$ of medium. This preparation was designated BIM.

The $C$. butyricum colonies on BIM had more irregular margins and were slightly smaller ( 1.5 to $3 \mathrm{~mm}$ in diameter) than colonies grown on VL medium.

Qualitative tests. The growth of 34 strains of $C$. butyricum, 10 strains of $C$. beijerinckii, 126 strains of other clostridia, 24 strains of non-spore-forming anaerobes, and 55 strains of various facultatively anaerobic bacteria on BIM was compared with that obtained on VL medium (Table 2). In addition to the 34 strains of $C$. butyricum and 10 strains of $C$. beijerinckii tested, all strains of other species of butyric acidproducing clostridia (BAPC) grew on BIM. The colonial appearance of most of these species was indistinguishable. Among the other clostridia tested, only $C$. sphenoides produced very small colonies on BIM. Among the 24 strains of non-spore-forming obligately anaerobic bacteria and the 55 strains of facultatively anaerobic bacteria tested, only Bacillus cereus grew on BIM. The B. cereus colonies on BIM were regularly circular and much smaller than the $C$. butyricum colonies on the same medium.

Quantitative tests. The quantitative recovery of two $C$. butyricum reference strains, seven $C$. butyricum isolates, three $C$. beijerinckii strains, and one Clostridium acetobutylicum strain was determined on VL, BM, and BIM (Table 3). Small differences between the number of colonies on the three media appeared for $C$. butyricum reference strains or isolates, and the $C$. beijerinckii and $C$. acetobutylicum recovery on BIM was low compared with that obtained on VL medium.

Recovery of $C$, butyricum from seeded fecal samples. Eight strains of $C$. butyricum, one strain of $C$. beijerinckii, and 12 fecal human samples were tested for recovery of $C$. butyricum or $C$. beijerinckii from seeded feces. The numbers of colonies recovered on BIM from seeded fecal samples usually were 10 to $100 \%$ of the number obtained on VL medium for $C$. butyricum strains, but not for the $C$. beijerinckii strain (Table 4). The recovery rate of $C$. beijerinckii
TABLE 1. Quantitative recovery of $C$. butyricum (CB 1002) on

$\mathrm{VL}$ medium and $\mathrm{BM}$ containing various concentrations of trimethoprim (TMP), cycloserine (Cycl), and polymyxin B ( $\mathrm{Pol} B$ )

\begin{tabular}{|c|c|c|c|c|c|}
\hline \multirow{2}{*}{ Medium } & \multicolumn{3}{|c|}{ Concn $(\mu \mathrm{g} / \mathrm{ml})$ of: } & \multirow{2}{*}{$\begin{array}{l}\text { No. of organisms } \\
\text { recovered per ml } \\
\text { of culture }\end{array}$} & \multirow{2}{*}{$\begin{array}{l}\text { No. of spores } \\
\text { recovered per } \\
\mathrm{ml} \text { of culture }\end{array}$} \\
\hline & TMP & Cycl & Pol B & & \\
\hline$\overline{\mathrm{VL}}$ & 0 & 0 & 0 & $1.75 \times 10^{6}$ & $4.7 \times 10^{7}$ \\
\hline \multirow[t]{7}{*}{$\mathbf{B M}^{a}$} & 0 & 0 & 0 & $1.39 \times 10^{6}$ & $4.6 \times 10^{7}$ \\
\hline & 16 & 10 & 10 & $1.26 \times 10^{6}$ & $2.7 \times 10^{7}$ \\
\hline & 16 & 10 & 20 & $1.15 \times 10^{6}$ & $2.8 \times 10^{7}$ \\
\hline & 16 & 10 & 30 & $0.70 \times 10^{6}$ & $\mathrm{ND}^{h}$ \\
\hline & 24 & 10 & 10 & $0.88 \times 10^{6}$ & ND \\
\hline & 24 & 10 & 20 & $0.51 \times 10^{6}$ & ND \\
\hline & 16 & 20 & 10 & $0.98 \times 10^{6}$ & ND \\
\hline
\end{tabular}

${ }^{a} \mathrm{BM}$ is a synthetic minimal medium without antimicrobial agents (see the text).

${ }^{b} \mathrm{ND}$, Not done.

from seeded feces was $1 \%$ of the cells present in the inoculum.

The BIM inhibited the growth of the fecal flora. Only a few colonies that were smaller than $0.5 \mathrm{~mm}$ were present on plates inoculated with 1:10 dilutions of fecal samples.

TABLE 2. Growth ${ }^{a}$ of various species of bacteria of BIM

\begin{tabular}{lrrr}
\hline \multicolumn{1}{c}{ Species } & $\begin{array}{c}\text { No. of } \\
\text { strains } \\
\text { tested }\end{array}$ & \multicolumn{2}{c}{$\begin{array}{c}\text { No. of strains } \\
\text { growing on: }\end{array}$} \\
\cline { 3 - 5 } & BIM & $\begin{array}{c}\text { VL } \\
\text { medium }\end{array}$ \\
\hline C. butyricum & 34 & 34 & 34 \\
C. beijerinckii & 10 & 10 & 10 \\
C. acetobutylicum & 21 & 21 & 21 \\
C. "butylicum" & 5 & 5 & 5 \\
C. pasteurianum & 1 & 1 & 1 \\
C. tyrobutyricum & 1 & 1 & 1 \\
C. "saccharoacetoperbutylaceticum" & 2 & 2 & 2 \\
C. sphenoides & 4 & $2^{b}$ & 4 \\
B. cereus & 6 & $5^{b}$ & 6 \\
\hline
\end{tabular}

a The strains that grew on VL control plates but not on BIM (number of tested strains) were: Clostridium absonum (1), C. aminovalericum (1), C. barkeri (1), C. bifermentans (2), C. botulinum A (1), C. botulinum D (1), C. botulinum E (1), C. cadaveris (1), C. carnis (1), C. clostridiforme (2), C. cochlearium (1), C. difficile (11), C. glycolicum (2), C. ghoni (2), C. histolyticum (1), C. innocuum (4), C. limosum (1), C. lituseburense (1), C. malenominatum (2), C. mangenotii (2), C. perenne (2), C. perfringens (14), C. putrificum (1), C. ramosum (1), C. rectum (1), C. sardiniensis (2), C. septicum (3), C. sordellii (13), C. sporogenes (2), C. sporosphaeroides (1), C. subterminale (2), C. tetani (2), C. tertium (4); Bacteroides bivius (1), B. fragilis (4), B. melaninogenicus (1), B. ureolyticus (1); Fusobacterium nucleatum (1), $F$. necrophorum (3), F. varium (1); Peptococcus magnus (1), Peptostreptococcus anaerobius (1), Peptococcus anaerobius (1); Propionibacterium acnes (3): Eubacterium lentum (1); Bifidobacterium species (5); Citrobacter species (1); Escherichia coli (3); Edwardsiella tarda (1); Enterobacter aerogenes (1), E. agglomerans (1), E. cloacae (1), E. hafniae (1); Klebsiella pneumoniae (1), K. oxytoca (1), K. ozaenae (1), K. rhinoscleromatis (1); Levinea amalonatica (1), L. malonatica (1); Proteus mirabilis (1), P. morganii (1), P. rettgeri (1), P. vulgaris (1); Providentia alcalifaciens (1), P. stuartii (1); Salmonella choleraesuis subsp. choleraesuis serovar oranienburg (1), S. cholerae-suis subsp. cholerae-suis serovar brandenburg (1), S. cholerae-suis subsp. cholerae-suis serovar typhimurium (1); Serrratia liquefaciens (1), S. marcescens (1); Bacillus cereus subsp. mycoides (1), B. coagulans (1), B. firmus (1), B. licheniformis (1), B. macerans (1), B. polymyxa (1), B. subtilis (1); Lactobacillus acidophilus (1), L. casei subsp. casei (1), L. casei subsp. rhamnosus (1), L. delbrueckii subsp. delbrueckii (1), L. fermentum (1), $L$. jensenii (1), $L$. delbrueckii subsp. lactis (1), L. plantarum (1), L. salivarius (1), L. viridescens (1); Streptococcus faecalis (1), S. faecalis subsp. liquefaciens (1), S. faecalis subsp. zymogenes (1), S. faecium (1), S. salivarius (1), S. pyogenes (1): Staphylococcus aureus (2), S. epidermidis (1), S. haemolyticus (1), S. hominis (1), S. saprophyticus (1).

${ }_{b}^{b}$ Small colonies. 
TABLE 3. Recovery of $C$. butyricum and other BAPC on VL, $\mathrm{BM}$, and BIM

\begin{tabular}{lccc}
\hline \multirow{2}{*}{ Strain } & \multicolumn{3}{c}{$\begin{array}{c}\text { No. of organisms recovered per ml of culture } \\
\text { on: }\end{array}$} \\
\cline { 2 - 4 } & VL & BM & BIM \\
\hline C. butyricum & & & \\
VPI 3266 & $6.6 \times 10^{6}$ & $2.0 \times 10^{6}$ & $1.0 \times 10^{6}$ \\
VPI 2969 & $7.9 \times 10^{6}$ & $1.3 \times 10^{6}$ & $2.1 \times 10^{6}$ \\
$153-2^{a}$ & $11.3 \times 10^{7}$ & $8.3 \times 10^{7}$ & $8.0 \times 10^{7}$ \\
$181-2^{a}$ & $8.7 \times 10^{6}$ & $3.0 \times 10^{6}$ & $2.0 \times 10^{6}$ \\
$147-1^{a}$ & $5.5 \times 10^{7}$ & $4.9 \times 10^{7}$ & $3.1 \times 10^{7}$ \\
$144-1^{a}$ & $1.5 \times 10^{7}$ & $3.1 \times 10^{6}$ & $5.0 \times 10^{6}$ \\
$174-3^{a}$ & $3.6 \times 10^{7}$ & $1.6 \times 10^{7}$ & $9.2 \times 10^{6}$ \\
$116-1^{a}$ & $0.9 \times 10^{8}$ & $1.3 \times 10^{8}$ & $1.1 \times 10^{8}$ \\
& & & \\
C. beijerinckii & & & \\
VPI 5481 $_{\text {VPI 2681 }}$ & $1.7 \times 10^{8}$ & $5.0 \times 10^{6}$ & $10^{5}$ \\
CNRZ 534 $^{8}$ & $1.7 \times 10^{8}$ & $1.2 \times 10^{8}$ & $10^{5}$ \\
C. acetobutylicum & $2.9 \times 10^{6}$ & $1.4 \times 10^{6}$ & $10^{5}$ \\
AB 23 & & & \\
\hline
\end{tabular}

"These $C$. butyricum isolates were isolated from newborn feces (P. Raibaud, Centre National de Recherches Zootechniques, Jouy-en-Josas, France).

\section{DISCUSSION}

The methods routinely used for isolation of $C$. butyricum from fecal samples are those described for most clostridia and include spore selection techniques by heat or alcohol treatment and the use of blood agar or egg yolk agar with neomycin and cycloserine (18) or with kanamycin (20). But these methods allow the growth of a wide variety of clostridia and other bacteria from fecal flora.

Cummins and Johnson (2) reported that $C$. butyricum but not $C$. beijerinckii strains grew on a basal glucose-mineral salts medium supplemented with biotin. Our observations showed that (i) both $C$. butyricum and $C$. beijerinckii strains grew on fluid BM, (ii) some $C$. beijerinckii strains produced colonies smaller than $C$. butyricum, and (iii) the quantitative recovery of $C$. beijerinckii strains on BIM was less efficient than that of $C$. butyricum strains (Tables 3 and 4). The use of

TABLE 4. Recovery of $C$. butyricum from seeded fecal specimens on BIM

\begin{tabular}{cccc}
\hline $\begin{array}{c}\text { Fecal } \\
\text { specimen } \\
\text { no. }\end{array}$ & $\begin{array}{c}\text { C. butyricum } \\
\text { strain }\end{array}$ & $\begin{array}{c}\text { No. of colonies } \\
\text { recovered from } \\
\text { fecal specimen } \\
\text { on BIM }\end{array}$ & $\begin{array}{c}\text { Size (CFU/ml) of } \\
\text { the } C \text {. butricum } \\
\text { inoculum used to pre- } \\
\text { pare seeded stools }\end{array}$ \\
\hline 1 & VPI 3266 & $8.0 \times 10^{3}$ & $6.2 \times 10^{3}$ \\
2 & VPI 2417 & $1.0 \times 10^{5}$ & $2.8 \times 10^{6}$ \\
3 & VPI 2969 & $8.0 \times 10^{4}$ & $6.2 \times 10^{4}$ \\
4 & VPI 2681 & $2.0 \times 10^{4}$ & $2.0 \times 10^{6}$ \\
5 & CB 174-3 & $5.9 \times 10^{3}$ & $11.3 \times 10^{3}$ \\
6 & CB 87-2 & $3.0 \times 10^{4}$ & $4.5 \times 10^{5}$ \\
7 & CB 116-1 & $2.3 \times 10^{4}$ & $3.2 \times 10^{4}$ \\
8 & CB 181-2 & $2.1 \times 10^{4}$ & $2.7 \times 10^{4}$ \\
9 & CB 1002 & $1.0 \times 10^{4}$ & $3.0 \times 10^{4}$ \\
10 & CB 1002 & $8.0 \times 10^{4}$ & $3.0 \times 10^{4}$ \\
11 & CB 1002 & $3.2 \times 10^{3}$ & $3.0 \times 10^{4}$ \\
12 & CB 1002 & $8.0 \times 10^{3}$ & $3.0 \times 10^{4}$ \\
\hline
\end{tabular}

a All colonies were considered to be $C$. butyricum, since unseeded specimens did not yield any colonies and all colonies tested produced the same microscopic appearance and the same pattern of sugar fermentation as $C$. butyricum.

${ }^{h}$ The $C$. butyricum cell suspension used for seed fecal specimens was diluted in FBM as a fecal specimen and inoculated on VL plates.

'C. beijerinckii. the BM allowed the growth of some BAPC, principally $C$. butyricum, and inhibited the growth of the proteolytic clostridia and other clostridia with more complex nutritional requirements.

The incorporation of cycloserine, trimethoprim, and polymyxin B in BM improved the selectivity of this medium. The cycloserine had previously been used for the isolation of Clostridium perfringens (6) and Clostridium difficile (21); the cycloserine-cefoxitine combination had been used for the isolation of $\dot{C}$. difficile (4); the cycloserine-trimethoprimsulfamethoxazole combination had been used for the isolation and enumeration of clostridia in human feces (14); and polymyxin associated with oleandomycin and sulfadiazin had been used for $C$. perfringens quantitation in foods $(5,6)$. Although many $C$. butyricum isolates from human sources produce a $\beta$-lactamase $(11,12), \beta$-lactam antibiotics would not be satisfactory for the isolation of those $C$. butyricum strains that do not produce a $\beta$-lactamase.

With BIM, some of the BAPC could be recovered from fecal specimens, whereas the majority of other fecal microorganisms were inhibited. Among the other bacteria, $B$. cereus strains and some $C$. sphenoides strains grew on BIM. The colonies of these organisms were circular and small and could be distinguished easily from BAPC colonies. No obvious differences were observed among the colonies of the different BAPC. However, $C$. butyricum is the main BAPC isolated from human feces (13) and is quantitatively recovered on BIM. If the number of $C$. butyricum organisms is low in fecal samples, fluid BIM could be used as enrichment medium before inoculation of BIM plates.

Our data also indicate that BIM, with some modifications, can be used for the isolation of some other BAPC, such as $C$. acetobutylicum. The recovery of BAPC from soil specimens was not attempted.

\section{ACKNOWLEDGMENTS}

We thank J. Morin for technical assistance and M. Sebald for helpful advice and criticism during the course of this work.

\section{LITERATURE CITED}

1. Beerens, H. 1954. Amelioration des techniques d'étude et d'identification des bactéries anaérobies. Ann. Inst. Pasteur de Lille 6:36-52.

2. Cummins, C. S., and J. L. Johnson. 1971. Taxonomy of the clostridia: wall composition and DNA homologies in Clostridium butyricum and other butyric acid-producing clostridia. J. Gen. Microbiol. 67:33-46.

3. Dezfulian, M., L. M. McCroskey, C. L. Hatheway, and V. R. Dowell, Jr. 1981. Selective medium for isolation of Clostridium botulinum from human feces. J. Clin. Microbiol. 13:526-531.

4. George, W. L., V. L. Sutter, D. Citron, and S. M. Finegold. 1979. Selective and differential medium for isolation of Clostridium difficile. J. Clin. Microbiol. 9:214-219.

5. Handford, P. M., and J. J. Cavett. 1973. A medium for the detection and enumeration of Clostridium perfringens (welchii) in foods. J. Sci. Food. Agr. 24:487.

6. Hauschild, A. H. W., and R. Hilsheimer. 1974. Enumeration of food-borne Clostridium perfringens in egg yolk-free tryptosesulfite-cycloserine agar. Appl. Microbiol. 27:521-526.

7. Holdeman, L. V., E. P. Cato, and W. E. C. Moore (ed.). 1977. Anaerobe laboratory manual, 4th ed. Virginia Polytechnic Institute and State University, Blacksburg, Va.

8. Howard, F. M., J. M. Bradley, D. M. Flynn, P. Noone, and M. Szawatkowski. 1977. Outbreak of necrotizing enterocolitis caused by Clostridium butyricum. Lancet ii:1099-1102.

9. Labbe, R. G. 1981. Enterotoxin formation by Clostridium perfringens type $\mathrm{A}$ in a defined medium. Appl. Environ. Microbiol. 41:315-317.

10. Laverdiere, M., A. Robert, R. Chicoine, D. Salet, and R. 
Rosenfeld. 1978. Clostridia in necrotizing enterocolitis. Lancet ii:377.

11. Magot, M. 1981. Résistance aux pénicillines dûes à la production d'une bêta-lactamase chez des Clostridium du groupe butyricum. C.R. Acad. Sci. 292:285-287.

12. Magot, M. 1981. Some properties of the Clostridium butyricum group B-lactamase. J. Gen. Microbiol. 127:113-119.

13. Magot, M., J. P. Carlier, and M. R. Popoff. 1983. Identification of Clostridium butyricum and Clostridium beijerinckii by gasliquid chromatography and sugar fermentation: correlation with DNA homologies and electrophoretic patterns. J. Gen. Microbiol. 129:2837-2845.

14. Mevissen-Verhage, E. A. E., N. M. De Vos, W. C. M. HarmsenVan Amerongen, and J. H. Marcelis. 1982. A selective medium for the detection and enumeration of clostridia in human feces. Antonie van Leevwenhoek J. Microbiol. Serol. 48:205-206.

15. Popoff, M. R., and M. Sebald. 1981. Mise en évidence chez Clostridium butyricum d'un facteur thermostable responsable du pouvoir pathogène expérimental. C.R. Acad. Sci. 292:763766.
16. Sebald, M., and R. N. Costilow. 1975. Minimal growth requirements for Clostridium perfringens and isolation of auxotrophic mutants. Appl. Microbiol. 29:1-6.

17. Skerman, V. B. D., V. Mc Gowan, and P. H. A. Sneath. 1980 Approved lists of bacterial names. Int. J. Syst. Bacteriol. 30:225-420.

18. Smith, M. F., S. P. Borriello, G. S. Clayden, and M. W. Casewell. 1980. Clinical and bacteriological findings in necrotizing enterocolitis: a controlled study. J. Infection 2:23-31.

19. Sturm, R., J. L. Staneck, L. R. Stauffer, and W. W. Nebleh. 1980. Neonatal necrotizing enterocolitis associated with penicillin resistant, toxigenic Clostridium butyricum. Pediatrics 66:928-931.

20. Westra-Meijer, C. M. M., J. E. Degrener, G. Dzoljic-Danilovic, M. F. Michel, and J. W. Mettau. 1983. Quantitative study of the aerobic and anaerobic foecal flora in neonatal necrotizing enterocolitis. Arch. Dis. Child. 58:523-528.

21. Willey, S. H., and J. G. Bartlett. 1979. Cultures for Clostridium difficile in stools containing a cytotoxin neutralized by Clostridium sordellii antitoxin J. Clin. Microbiol. 10:880-884. 